\title{
IMPLICANCIAS DE LA CAPACITACIÓN VIRTUAL EN LOS GOBIERNOS LOCALES
}

Yolanda Bustamante Sánchez*

E-mail:yobusa22@yahoo.es,ybustamantes@unmsm.edu.pe

\section{RESUMEN}

Los gobiernos locales en el Perú tienen una función importante en el proceso de descentralización y desarrollo del país. No obstante, existe una falta de programas de capacitación para los recursos humanos en estas instituciones lo que imposibilita proponer proyectos sociales. Por esta razón, es necesario promocionar cursos virtuales a distancia en diversas áreas como Gestión Pública, Desarrollo Humano, Participación Ciudadana, Estrategias para la Gestión de Políticas Intergubernamentales, Administración Pública, entre otros.

Palabras clave: Capacitación virtual, gobiernos locales, aprendizaje, descentralización, gestión.

\begin{abstract}
Local governments in Peru have an important function in the decentralization process and the development of the country. However, there is a lack of real training programs for human resources in these institutions what make them unable to propose social projects. For this reason, it is necessary to promote long-distance (virtual) training courses on areas such as Public Management, Human Development, Citizenship Participation, Strategies for Managing Intergovernmental Policies, Public Administration.
\end{abstract}

Key words: Virtual training, Local Governments, Learning, decentralization, management.

* Licenciada en Educación. Egresada de la Maestría en Administración - Recursos Humanos de la UNMSM. Especialización en Tecnología Educativa, Diplomado en Gestión Comercial Estratégica. Profesora de la Facultad de Ciencias Administrativas y Coordinadora del Programa de Idiomas Extranjeros de la Escuela de Postgrado de la Universidad Nacional Mayor de San Marcos. Actualmente está cursando un Diplomado de Gestión y Desarrollo Local en la Unión Iberoamericana de Municipalistas de Granada, España. 


\section{INTRODUCCIÓN}

En nuestro país, los Gobiernos Locales tienen un rol destacado en el proceso de descentralización y desarrollo integral; sin embargo, tienen múltiples deficiencias que les impide convertirse en agentes promotores del desarrollo local. La carencia de personal calificado, la escasa experiencia municipal de las autoridades, la falta de programas de capacitación, el desconocimiento del desarrollo de proyectos son, entre otros, los problemas que deben superar para convertirse en instituciones acordes con la exigencia de la modernidad. Es necesario, pues, contribuir con el desarrollo integral de las municipalidades para que puedan cumplir con sus objetivos a cabalidad.

\section{LA CAPACITACIÓN Y SU IMPORTANCIA}

La capacitación para el desarrollo de capacidades es un espacio de ínter aprendizaje e intercambio de información, que se convierte en un medio eficaz para hacer productivas a las personas. Esta capacitación puede ser impartida mediante lecturas, talleres vivenciales o a distancia, con el objetivo principal de proporcionar conocimientos en los aspectos técnicos del trabajo.

La capacitación incrementa las aptitudes y los conocimientos del trabajador con el propósito de prepararlo para que desempeñe eficientemente una unidad de trabajo específico y personal. Una capacitación a largo plazo permitirá el desarrollo del hombre, quien no sólo adquirirá conocimientos sino que logrará el fortalecimiento de su voluntad, la disciplina de carácter y la adquisición de las habilidades objetivo de cada curso.

Los tipos de capacitación son diversos:

- Por su naturaleza:

- Capacitación de orientación o de familiarización de los nuevos trabajadores con la organización (inducción).

- Capacitación vestibular o procedimiento simulado en el trabajo mismo.

- Capacitación en el trabajo o práctica en el mismo.

- Entrenamiento de aprendices o período formal de aprendizaje de un oficio.

- Entrenamiento técnico o tipo especial de preparación técnica del trabajador.
- Capacitación de supervisores que preparan al personal de supervisión para el desempeño de funciones gerenciales.

- Por el nivel ocupacional:

- Capacitación de operarios.

- Capacitación de obreros calificados.

- Capacitación de supervisores.

- Capacitación de jefes de línea.

- Capacitación de gerentes.

- Por la distancia

- Capacitación tradicional o presencial.

- Capacitación virtual.

\section{LA CAPACITACIÓN VIRTUAL}

Hoy en día, las tecnologías de la información y comunicaciones nos ofrecen muchas posibilidades para aprovechar sus recursos, tales como el Internet en donde se van a estructurar actividades de enseñanza-aprendizaje a través de recursos de contenidos (que permiten obtener información y que se da a través de lecciones, materiales, glosarios); recursos de comunicación (tales como foros, chat o charlas para expresar opiniones y debatir con el profesor y demás participantes); y recursos de ejercitación y evaluación (ejercicios sobre los temas del curso, calificación y comentarios sobre los progresos realizados en el proceso de aprendizaje a través de los diarios, tareas, consultas, encuestas, control de asistencias y participación).

La capacitación virtual es un tipo de enseñanza on line accesible a todos en un formato sincrónico (en tiempo real) o asincrónico (sin coincidencia temporal), en donde se dan un conjunto de actividades en red, que hacen posible la actualización de habilidades y el aprendizaje permanente de nuevas prácticas, gracias al uso de computadoras interconectadas entre sí.

El desafío de la capacitación virtual reside en cambiar el paradigma del aprendizaje, en modificar conductas establecidas y en realizar el autoaprendizaje estableciéndose espacios y tiempos para este fin.

Tiene como componentes al docente quien transmite los conocimientos y motiva el aprendizaje autónomo del alumno (agente activo), en una comunicación mediada a partir de tres fuentes:

- El contenido de los materiales elaborados didácticamente. 
- Las acciones tutoriales que motivan y ayudan al estudiante en su aprendizaje.

- El trabajo personal y colaborativo con otros estudiantes en grupos presenciales o en aulas virtuales.

\section{GOBIERNOS LOCALES Y TEMAS DE CAPACITACIÓN}

Los gobiernos locales son entidades básicas de la organización territorial del Estado y canales inmediatos de participación vecinal en los asuntos públicos, con autonomía política, económica y administrativa, que institucionalizan y gestionan con autonomía los intereses propios de las correspondientes colectividades, siendo elementos esenciales del gobierno local, el territorio, la población y la organización.

Las competencias y funciones específicas generales de los gobiernos locales son diversas y comprenden:

1. Planificar el desarrollo local y el ordenamiento territorial en el nivel provincial.

2. Promover la coordinación estratégica de los planes integrales de desarrollo distrital.

3. Promover, apoyar y ejecutar proyectos de inversión y servicios públicos municipales.

4. Emitir normas técnicas generales en materia de organización del espacio físico y uso del suelo, así como protección y conservación del ambiente.

5. Protección y conservación del ambiente.

6. En materia de desarrollo y economía local.

7. En materia de participación vecinal.

8. En materia de servicios sociales locales.

9. Prevención, rehabilitación y lucha contra el consumo de drogas.

Dentro del punto 4 se desprenden varias subactividades:

- Organización del espacio físico.

- Servicios públicos locales.

- Saneamiento ambiental, salubridad y salud

- Tránsito, circulación y transporte público

- Educación, cultura, deporte y recreación, donde los gobiernos locales tiene competencias y funciones específicas compartidas con el gobierno nacional y regional, vinculadas a promover el desarrollo humano; diseñar, ejecutar y evaluar el proyecto educativo de su jurisdicción; monitorear la gestión pedagógica, administrativa de las instituciones educativas bajo su jurisdicción; apoyar la creación de redes educativas; impulsar y organizar el Consejo Participativo Local de Educación; apoyar la incorporación y el desarrollo de nuevas tecnologías para el mejoramiento del sistema educativo; y organizar y sostener centros culturales, bibliotecas, teatros y talleres en la jurisdicción.

- Programas sociales, defensa y promoción de derechos ciudadanos.

- Seguridad ciudadana.

- Abastecimiento y comercialización de productos y servicios.

- Registros civiles.

- Promoción del desarrollo económico.

Se habla del Aprendizaje Continuo (longlife learning), entendido como el aprendizaje a lo largo de nuestra vida, que hoy en día resulta una realidad ya que los conocimientos fluyen y se actualizan constantemente, lo que obliga a estar actualizado y a retroalimentarse continuamente.

La Capacitación Virtual permite fortalecer las capacidades de los participantes de los gobiernos locales a fin de puedan gestionar el desarrollo integral de sus comunidades en temas varios, tales como procesos de planificación y gestión del desarrollo local; Estado, municipios y descentralización; metodologías de planificación estratégica; gestión municipal, proyectos de desarrollo, entre otros.

Si pensamos en realizar una capacitación virtual en los gobiernos locales proponemos que ésta debe tener los siguientes objetivos:

- Integrar a los elementos de los gobiernos locales responsables de la buena marcha de sus localidades, a fin de fortalecer sus capacidades de gestión.

- Actualizar los conocimientos de los participantes sobre los nuevos escenarios y tendencias de las políticas de las ciudades.

- Mejorar el rol de los administradores locales en el desarrollo de sus comunidades.

- Brindar instrumentos de gestión y su aplicación con eficiencia y eficacia en las comunidades locales. 


\section{IMPLICANCIAS DE LA CAPACITACIÓN VIRTUAL EN LOS GOBIERNOS LOCALES}

Una acertada capacitación virtual, a través de los medios que ofrecen las tecnologías de la información y comunicaciones en los gobiernos locales, va a incidir acertadamente en la gestión de las comunidades locales, para optimizar su rol de colaboradores directos del gobierno central en temas de gobernabilidad, planificación, dirección y control.

Las implicancias que de aquí se derivan son:

- Lograr la participación de todos los recursos humanos sin importar las distancias geográficas que los separan ni la coincidencia temporal de participantes y docentes, por lo que los participantes pueden tomar las clases durantes las 24 horas del día, en el momento más adecuado.

- Posibilitar la interacción de los docentes y sus compañeros en un aula virtual en donde el alumno tiene acceso a la clase orientadora del docente sobre los contenidos, así como a las propuestas de actividades a realizar y participar en los foros públicos

- Afirmar valores que promueven la autonomía, autoformación y desarrollo de capacidades para la gestión de desarrollo personal; voluntad, disciplina del carácter, independencia o grado de libertad para dirigir el proceso de aprendizaje; la competencia que implica el desarrollo de habilidades intelectuales y de actitudes para poder aprender de modo independiente, y apoyo que lo constituyen los recursos humanos y materiales que facilitan el aprendizaje.

- Fomentar la calidad de gestión de las organizaciones, en el desarrollo local y regional.

- Permitir el manejo óptimo del tiempo gracias a las tecnologías de la información, herramientas integradoras que permiten la comunicación directa y estructurada por ordenador (correo electrónico, listas de distribución, grupos de discusión, etc.), los teledebates como entorno de trabajo virtual sobre un tema concreto mediante el cual se envían mensajes de un tema exponiendo opiniones a favor o en contra, y la videoconferencia y chat en los que la comunicación se realiza en tiempo simultáneo y espacios diferidos. Las bases de datos telemáticas permiten que los alumnos puedan acceder a fuentes de información real y navegar en busca de información para el desarrollo de una tarea determinada.

- Incentivar el trabajo colaborativo a través de una visión compartida a fin de afrontar los retos produciendo la sinergia, ayudándose entre sí, complementando esfuerzos, compartiendo responsabilidades, favoreciendo la construcción de proyectos de innovación, promoción y desarrollo de los gobiernos locales.

\section{BIBLIOGRAFÍA}

Andía Valencia, Walter (2005). Gestión Pública. Lima, Centro de Investigación y Capacitación Empresarial (CICE), 293 pp.

Béjar, Héctor (2004). Justicia social, política social. Lima, CEDEP, 474 pp.

Escuela Mayor de Gestión Municipal (2004). Módulos 1, 2, 3. Lima, Imprensa S.R.L., 180 pp.

Garrison, D.R. y Anderson, Ferry (2003). ELearning in the $21^{\text {st }}$ century. USA, RoutledgerFalmer - Taylor \& Francis Group, 167 pp.

Geilius, Frans (1997). 80 herramientas para el desarrollo participativo. San Salvador, El Salvador, EDICPSA, 187 pp.

Katsioloudes, Marios I. (2002) Global stragetic planning: cultural perspectives for profit and nonprofit organizations. ButterworthHeinemann, 396 pp.

Margulis, Alexander (2002). Be in charge, a leadership manual. Academic Press. 118 pp.

Thornbury, Jan (2000). Living culture, a valuesdriven approach to revitalising your company culture. Random House, 188 pp.

Tiffin, John y Rajasingham, Lalita (1997). En busca de la clase virtual. Buenos Aires, Edit. Paidós, $274 \mathrm{pp}$. 